\title{
Optical study on relaxation of excitons and initiation of photopolymerization in diacetylene crystals at low temperatures
}

\author{
Chihiro Itoh \\ Department of Physics, Nagoya University, Furo-cho, Chikusa, Nagoya 464-8602, Japan \\ Present address: Department of Material Science and Chemistry, Wakayama University, \\ 930 Sakaedani, Wakayama 640-8510, Japan
}

\begin{abstract}
Luminescence and optical absorption induced by irradiating with a pulse of $266 \mathrm{~nm}$ laser beam have been measured in 5,7-dodecadiyne-1,12-diol bis[phenyl carbamate] (DA-TCDU). In addition to the recombination luminescence of the lowest triplet state, luminescence from the excited single state is identified. Irradiation with a pulse of $266 \mathrm{~nm}$ laser beam having the fluence above $4.4 \mathrm{mJcm}^{-2}$ leads to formation of series of optical absorption bands at $3.05 \mathrm{eV}, 2.52 \mathrm{eV}$ and $2.18 \mathrm{eV}$, which are ascribed to the optical transition due to dimer diradicals, trimer diradicals and the tetramer diradicals, respectively. The laser-fluence dependence of the integral intensity of the recombination luminescence of the singlet and the triplet exciton and the absorption strength of the $3.05 \mathrm{eV}$ band have been measured. The nature of the triplet exciton and its relation to the initiation of the photopolymerization are discussed.
\end{abstract}

\section{INTRODUCTION}

Photoexcitation of organic crystals often leads to change of the molecular configuration or chemical reactions, which generally resemble those of molecules in solutions. However, it is known that there are exotic crystals which exhibit photochemical reactions distinct from that found in solutions. Photopolymerization of crystalline diacetylenes $\left(\mathrm{R}-\mathrm{C} \equiv \mathrm{C}-\mathrm{C} \equiv \mathrm{C}-\mathrm{R}^{\prime}\right)$ is a typical example of such reaction [1, 2]. Irradiation of single crystals of diacetylenes with UV light or gamma ray triggers solid-state polymerization, and the monomer crystals transform into polydiacetylene crystals with nearly the same dimensions as the monomer crystals. This Photopolymerization has attracted considerable interest, and extensive studies have been carried out for revealing its mechanism [3]. In recent years, there has been renewal of interest in this photopolymerization as an example of photo-induced cooperative phenomena, and theoretical studies have been made in terms of photo-induce phase transition in solids [4]. On the basis of the insights accumulated so far, photopolymerization is initiated by formation of dimer diradicals, and the formation of stable polymer chain follows successive addition reaction of reaction intermediates along the stacking axis. Although the configuration of the reaction intermediates formed during the polymerization have been studied in details, only limited insight on the dimer-diradical formation has been accumulated. The important finding on dimer-diradical formation have been made by the Neumann and Sixl [5]. They showed that the EPR signal originated from the reaction intermediates induced by irradiating with UV light depends nonlinearly on the illumination dose. Because of the result, they proposed that the dimer diradical is formed as the result of bimolecular reaction of the lowest triplet states $[5,6]$. However, this suggestion has not been validated experimentally. This is partly because only limited knowledge on the lowest triplet state in diacetylene crystals has been accumulated. Thus, elucidation of the characteristics of the lowest triplet state including the relation to the dimer-diradical formation are required for understanding the mechanism of the dimer-diradical formation.

Recently, Itoh, Kondoh and Tanimura [7] have found that a broad luminescence band having a peak at $2.57 \mathrm{eV}$ is induced by irradiation of single crystal of 5,7-dodecadiyne-1,12-diol bis[phenyl carbamate] (DATCDU; $\left.\mathrm{R}=\mathrm{R}^{\prime}=\left(\mathrm{CH}_{2}\right)_{4} \mathrm{O}(\mathrm{CO}) \mathrm{NHC}_{6} \mathrm{H}_{5}\right)$ with a pulse of $1 \mathrm{MeV}$ electron beam at $10 \mathrm{~K}$. They found that the $2.57 \mathrm{eV}$ luminescence decays single exponentially with a time constant of $113 \mathrm{~ms}$ at $10 \mathrm{~K}$. The authors attributed the $2.57 \mathrm{eV}$ luminescence to the radiative recombination of the lowest triplet state because of its long decay.

In this paper, the experimental results on the measurements of the luminescence and the optical absorption induced in DA-TCDU are presented. The nature of excited states induced by optical excitation and the mechanism of the dimer-diradical formation induced are discussed.

\section{MATERIALS AND METHODS}

DA-TCDU powder obtained from Dojindo, co. LTD. was purified by repeated recrystalization from acetone solutions. Single crystals of DA-TCDU with typical dimensions of $3 \times 3 \times 1 \mathrm{~mm}^{3}$ were obtained from acetone solutions by slow evaporation at 25 centigrade. The crystals thus obtained were transparent and showed no detectable optical absorption in the wavelength range from $800 \mathrm{~nm}$ to $285 \mathrm{~nm}$ at room temperature. Specimen, which was prepared by cleaving from the as-grown crystal, was attached at cold finger of a conventional 
cryostat, and it cooled down to $8 \mathrm{~K}$. For exciting the specimens, $266 \mathrm{~nm}$ laser pulses generated by laser system comprised from a pulsed YAG:Nd laser (Coherent Infinity 40) operated at a repetition of $10 \mathrm{~Hz}$ and a BBO crystal for converting second harmonic of the YAG laser beam into fourth harmonic was employed. The time duration of the laser pulse is $5 \mathrm{~ns}$, and the repetition ratio of the excitation was controlled in the range from $10 \mathrm{~Hz}$ to $1 \mathrm{~Hz}$ using a mechanical shutter placed in the excitation path. The luminescence spectra induced by irradiating with laser pulses were measured by the multichannel detection system, which consists of a monochrometer (Jobin Yvon HR-320) and a gatable multichannel detector (Princeton IRY-1024). For measuring the integral intensity of the luminescence, the luminescence from the specimen was guided to the optical detection system comprised from a monochrometer (Jobin Yvon CP-200) and a CCD detecor (Princeton TE/CCD-1100PF). Optical absorption spectra induced following the laser irradiation were measured using a flush Xe lamp (Hamamatsu L2358) as the light source and the detection system which was the same as that used for measuring the integral intensity of the luminescence.

\section{EXPERIMENTAL RESULTS}

Luminescence spectra induced by irradiating with a pulse of $266 \mathrm{~nm}$ laser beam having the fluence lower than $0.5 \mathrm{mJcm}^{-2}$ at $8 \mathrm{~K}$ are shown in Figure 1 . The luminescence band at $4.26 \mathrm{eV}$ was observed prominently at the time delay of $2 \mathrm{~ns}$ with a gate width of $1 \mathrm{~ns}$. A broad luminescence band having a peak at $2.57 \mathrm{eV}$ was measured at $10 \mathrm{~ms}$ with a gate width of $8 \mathrm{~ms}$. By the luminescence-intensity measurements at various delay time from the excitation with the fixed gate width, the $4.26 \mathrm{eV}$ and the $2.57 \mathrm{eV}$ luminescence were found to decay with a time constant of about $5 \mathrm{~ns}$ and about $100 \mathrm{~ms}$, respectively. The spectrum of the $2.57 \mathrm{eV}$ luminescence is identical with that induced by irradiation with a $1 \mathrm{MeV}$ electron pulse, and the luminescence intensity was reported to decay single exponentially with a time constant of $113 \mathrm{~ms}$ [7].

Optical absorption spectra of DA-TCDU crystal have measured after $60 \mathrm{~s}$ following the irradiation with a pulse of $266 \mathrm{~nm}$ laser beam having various fluences, and the results are shown in Figure 2. Each spectrum shown in the figure was measured by changing the spot at every irradiation. At the fluence of $4.4 \mathrm{mJcm}^{-2}$, weak absorption exhibiting a peak at $3.05 \mathrm{eV}$ was induced. Irradiation with larger fluence gives rise to growth of series of absorption bands at $2.52 \mathrm{eV}$ and $2.18 \mathrm{eV}$ in addition to the $3.05 \mathrm{eV}$ absorption. Similar series of the sharp absorption bands have been reported in several diacetylene crystals, and the bands have been assigned to optical transition of the reaction intermediates having diradical form [8]. As rising the sample temperatures, reduction in the absorption strength of the $3.05 \mathrm{eV}$ band and increase of the $2.52 \mathrm{eV}$ band were observed. This clearly shown that the $2.52 \mathrm{eV}$ band is

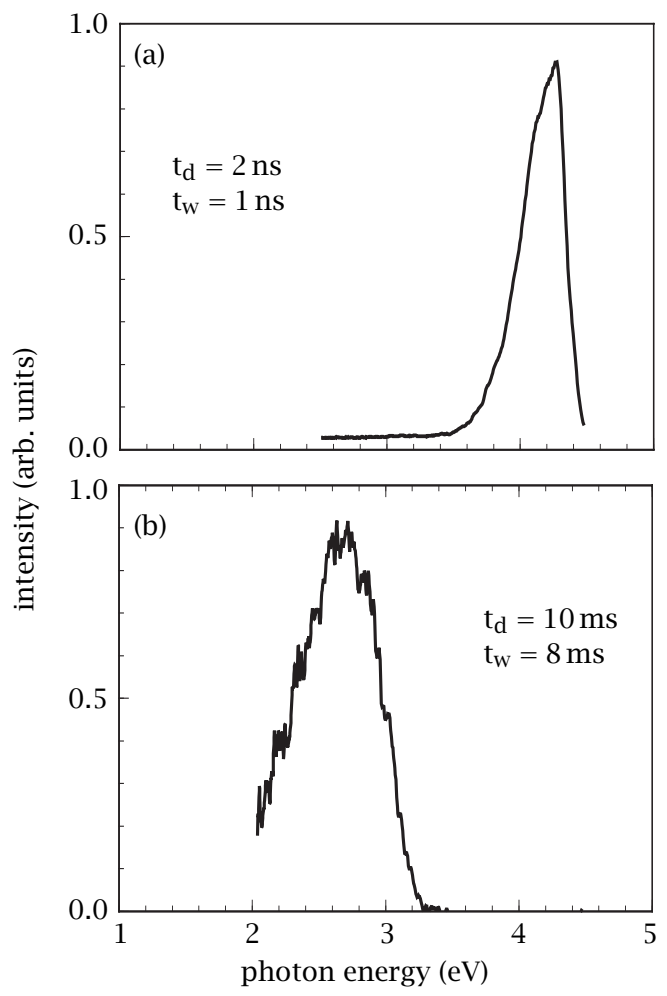

Figure 1. Luminescence spectra of DA-TCDU crystals measured at the delay time $\left(\mathrm{t}_{\mathrm{d}}\right)$ of $5 \mathrm{~ns}$ with the gate width $\left(\mathrm{t}_{\mathrm{w}}\right)$ of $2 \mathrm{~ns}(\mathrm{a})$ and at $\mathrm{t}_{\mathrm{d}}=10 \mathrm{~ms}$ with $\mathrm{t}_{\mathrm{w}}=8 \mathrm{~ms}$ (b) following the irradiation with a pulse of $266 \mathrm{~nm}$ laser beam at $8 \mathrm{~K}$.

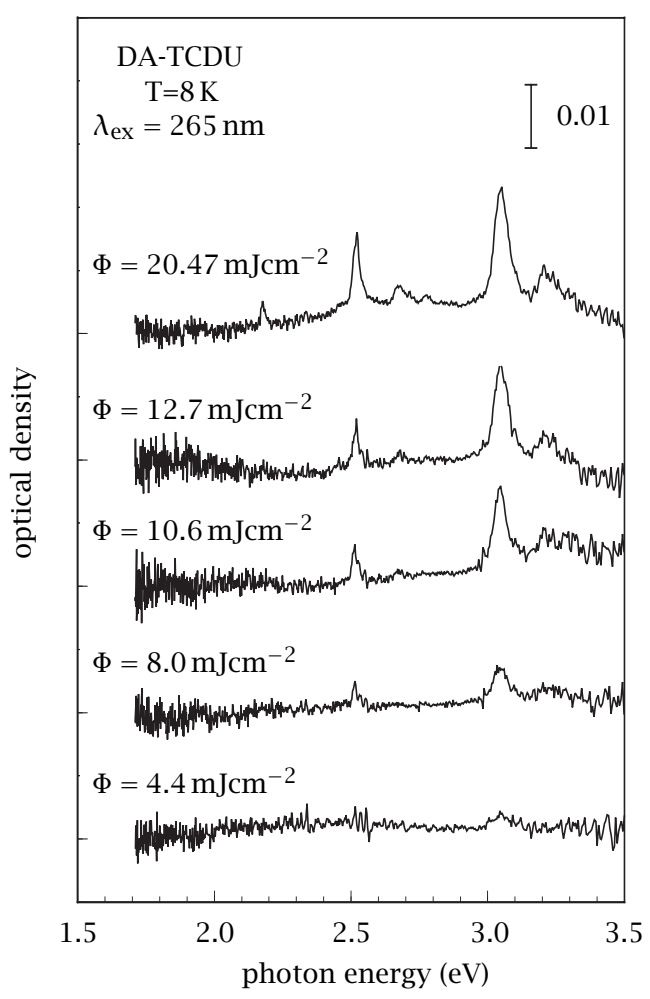

Figure 2. Optical absorption spectra of DA-TCDU crystals measured after $60 \mathrm{~s}$ following the $266 \mathrm{~nm}$ laser excitation with various fluences at $8 \mathrm{~K}$. 
formed at the expense of the $3.05 \mathrm{eV}$ band. The $3.05 \mathrm{eV}$, the $2.52 \mathrm{eV}$ and the $2.18 \mathrm{eV}$ absorption are, therefore, attributed to optical transition due to dimer diradicals, trimer diradicals and the tetramer diradicals, respectively.

In order to get detailed information on the dimerdiradical formation, the fluence-dependence of the integral intensity of the $4.26 \mathrm{eV}$ and the $2.57 \mathrm{eV}$ luminescence have been measured together with that of the peak strength of the $3.05 \mathrm{eV}$ absorption induced following the excitation. The results are plotted as a function of laser fluence in Figure 3. It is clear that the absorption strength of the $3.05 \mathrm{eV}$ band increases nonlinearly with increasing the laser fluence. This result was consistent with the previous report [5]. On the other hand, the integral intensity of the $4.26 \mathrm{eV}$ and the $2.57 \mathrm{eV}$ luminescence show distinct behavior in this fluence region. While the integral intensity of the $4.26 \mathrm{eV}$ luminescence is in proportion with the laser, the $2.57 \mathrm{eV}$ luminescence exhibits saturation at higher fluences. Deviation from the linear increase of the $2.57 \mathrm{eV}$ luminescence rises in the fluence region where the $3.05 \mathrm{eV}$ absorption shows marked increase. The result implies that the dimer-diradical formation is in coompetition with the emission of the $2.57 \mathrm{eV}$ luminescence.

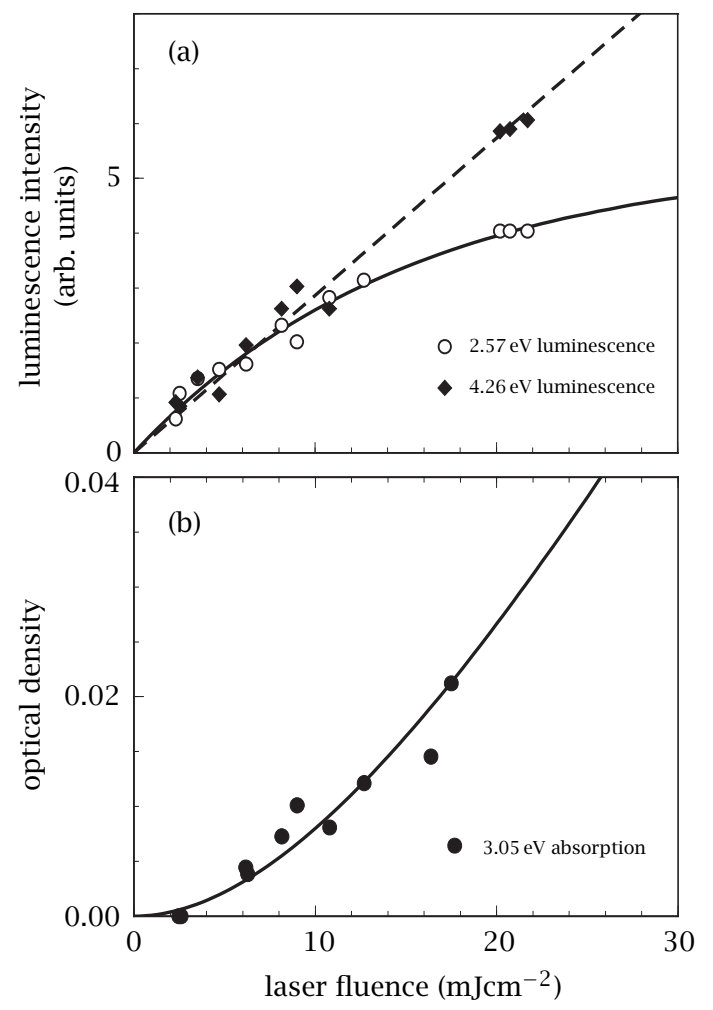

Figure 3. Laser-fluence dependence of the integral intensity of the $2.57 \mathrm{eV}$ and the $4.26 \mathrm{eV}$ luminescence (a) and the strength of the $3.05 \mathrm{eV}$ absorption band (b) induced by irradiating with a pulse of $266 \mathrm{~nm}$ laser beam in DA-TCDU crystal at $8 \mathrm{~K}$. Solid and broken curves shown in the figure are the results of the parameter fitting (see the text).

\section{DISCUSSION}

Excitation of DA-TCDU crystal with a pulse of $266 \mathrm{~nm}$ laser beam induces the $4.26 \mathrm{eV}$ and the $2.57 \mathrm{eV}$ luminescence bands. In terms of the decay time, it is reasonable to ascribe these bands to radiative transition from the singlet and the triplet state, respectively. Recently, the excitation spectra for the $4.26 \mathrm{eV}$ and the $2.57 \mathrm{eV}$ luminescence have been measured [9], and the results demonstrate that these luminescence are induced by iluminating with UV phonons having an energy above the onset of the fundamental absorption of DA-TCDU. On the basis of this result, these luminescence are attributed to radiative recombination of the intrinsic excited states. The $4.26 \mathrm{eV}$ and the $2.57 \mathrm{eV}$ luminescence bands are, therefore, ascribed to radiative recombination of the singlet and the triplet excitons, respectively.

It is notable that the $2.57 \mathrm{eV}$ luminescence shows single exponential decay. In organic crystals in which exciton has large mobility, like anthracene, decay of the recombination luminescence is governed by collision of excitons but not by monomolecular deexcitation. In such crystals, the triplet exciton generally decays nonexponentially because of this bimolecular process. The single exponential decay of the triplet-exciton luminescence is a manifestation that the deexcitation of the triplet excitons in DA-TCDU crystals is governed by monomolecular process. This implies that the triplet exciton in DA-TCDU crystal has limited mobility. Low mobility of the triplet exciton is also suggested by another characteristics of the $2.57 \mathrm{eV}$ luminescence: the broad and unstructured spectrum and the large peak shift of $1.71 \mathrm{eV}$ from the onset of the fundamental absorption. These features imply that the initial state of this luminescence is accompanied with large lattice relaxation. The existence of the large lattice relaxation has been also elucidated by the measurement of luminescence polarization [7]. Large lattice relaxation accompanied by the lowest triplet state leads to localization of the lowest triplet state because of breaking of the transnational symmetry in the crystal. Consequently, the $2.57 \mathrm{eV}$ luminescence is conceivably originated from the localized triplet center with large lattice relaxation formed by the relaxation of intrinsic excitation. Such a metastable center is known as self-trapped exciton, which is well established in inorganic and organic crystals [10].

The nonlinear increase of the yield of dimer diradical implies that its formation involves interaction of the excited species, as suggested previously, but not simple monomolecular relaxation of the excited state. In the fluence region in which the dimer diradical is formed efficiently, the $2.57 \mathrm{eV}$ luminescence shows underlinear increase, while the integral intensity of the $4.26 \mathrm{eV}$ luminescence is in proportion with the laser fluence. Based on the results, it is clear that the dimer-diradical formation is in competition with radiative recombination of the self-trapped excions. The model that dimerdiradical formation involves bimolecular reaction of the lowest triplet state may not conflict with the experimen- 
tal result obtained in this study. However, the possibility of the formation of a pair of the lowest triplet states at adjacent sites is conceivably zero at low temperature. This is because, as discussed above, the lowest triplet state in DA-TCDU crystal is regarded as the self-trapped excitons, whose mobility is vanished at low temperature due to large lattice relaxation. Therefore, the model involving bimolecular reaction of the selftrapped excitons is not appropriate for explaining the dimer-diradical formation.

The alternative model has been suggested by Itoh, Kondoh and Tanimura very recently [11]. In this model, the dimer-diradical formation involves collision of unrelaxed excitons with the self-trapped excitons. For examining the model, the experimental results are analyzed in terms of the kinetic equation model as the followings. Irradiation of a laser pulse having flux of $\phi$ generates unrelaxed excitons at efficiency of $\sigma$. The exciton thus generated relaxes into the excited singlet and the lowest triplet state by the rate of $\gamma_{i}(i=S$ or $T)$ respectively. The dimer diradicals are formed at efficiency of $\eta$ by collision of unrelaxed excitons with the lowest triplets. The kinetics of this system are described by following equations:

$$
\begin{aligned}
\frac{d N_{\mathrm{EX}}}{d t} & =\sigma \phi-\sum_{i} \gamma_{i} N_{\mathrm{EX}}-\eta N_{T} N_{\mathrm{EX}}, \\
\frac{d N_{T}}{d t} & =\gamma_{T} N_{\mathrm{EX}}-\lambda_{i} N_{T}-\eta N_{T} N_{\mathrm{EX}}, \\
\frac{d N_{S}}{d t} & =\gamma_{S} N_{\mathrm{EX}}-\lambda_{S} N_{S}, \\
\frac{d N_{P}}{d t} & =\eta N_{T} N_{\mathrm{EX}},
\end{aligned}
$$

wher $N_{i}(i=E X, S, T$ or $D)$ represents the population of the unrelaxed excitons, the singlet excitons, the selftrapped excitons and dimer diradicals, respectively. In these equations, $\lambda_{i}$ denotes a monomolecular deexcitation rate of the excited state labeled $i$. In the experimental condition employed in this study, relaxation of the exciton generated by optical excitation is considered much faster than the generation given by the rate of $\sigma \phi$. Typical decay time of the unrelaxed exciton in organic crystals is considered several pico second, which is much shorter than the time duration of the excitation pulse employed in this study. In this condition, the population of the unrelaxed excitons is conceivable in steady state within the duration of the excitation pulse. In the low laser-fluence regime, it is conceivable that relaxation of the unrelaxed excitons is conceivably faster than the generation of dimer diradicals. Thus, for simplifying the kinetic equations, the case where $\sum_{i} \gamma_{i} \gg \eta N_{T}$ is considered. Then the populations of the unrelaxed exciton, the singlet exciton, the triplet selftrapped exciton and the dimer diradicals induced after the excitation are given by

$$
\begin{aligned}
& N_{\mathrm{ex}}=\sigma \phi \sum_{i} \gamma_{i} \\
& N_{T}=\frac{\omega_{T} \sigma \phi}{\lambda_{T}+\omega_{D} \sigma \phi}\left[1-\exp \left\{\left(\lambda_{T}+\omega_{D} \sigma \phi\right) \tau\right\}\right],
\end{aligned}
$$

$$
\begin{aligned}
N_{S} & =\omega_{S} \sigma \phi\left[1-\exp \left(-\lambda_{S} \tau\right)\right], \\
N_{D} & =\omega_{D} \omega_{T}\left(\frac{\sigma \phi}{\lambda_{T}+\omega_{D} \sigma \phi}\right)^{2} \\
\cdot & {\left[\left(\lambda_{T}+\omega_{D} \sigma \phi\right) \tau+\exp \left\{\left(\lambda_{T}+\omega_{D} \sigma \phi\right) \tau\right\}-1\right],(8) } \\
\omega_{j} & =\frac{\gamma_{j}}{\sum_{i} \gamma_{i}}(j=T, S), \quad \omega_{D}=\frac{\eta}{\sum_{i} \gamma_{i}},
\end{aligned}
$$

where $\tau$ is the time duration of the laser pulse. The integral intensity of the recombination luminescence of the triplet self-trapped exciton and the singlet exciton are in proportion with their population given by the equations (6) and (7). The equations (6) and (7) were fitted to the data points shown in Figure 3(a) first. The solid and broken curves shown in Figure 3(a) are the results of the parameter fitting of the equations (6) and (7) and the data points. The curves well explain the laserfluence dependence of the intensity of the $4.26 \mathrm{eV}$ luminescence and the $2.57 \mathrm{eV}$ luminescence. It is notable that the equation (8) with the same parameter set obtained by this fitting can reproduce the laser-fluence dependence of the dimer-diradical yield with a parameter for adjusting the magnitude as shown by the solid curve in Figure 3(b). The good agreement of the fitting curves to the experimental points implies that the model proposed here is appropriate for explaining the photo-induced dimer-diradical formation. The fitting, however, gives only qualitative interpretation on the experimental results, because the parameters required for quantitative discussion, like oscillation strength of the $3.05 \mathrm{eV}$ absorption, have not been obtained yet. For elucidating the microscopic mechanism of the photoinduced dimer-diradical formation, further studies on the relaxation dynamics at excited states in diacetylene crystals with higher time-resolution are required.

\section{ACKNOWLEDGEMENTS}

The author is grateful to Prof. K. Tanimura and Mr. T. Kondoh for many valuable discussions.

\section{REFERENCES}

[1] G. Wegner, Makromol. Chem. 145 (1971), 85.

[2] G. Wegner, Pure Appl. Chem. 154 (1972), 35.

[3] H. J. Catlow (ed.), Advances in polymer science, vol. 63, Polydiacetylenes Springer-Verlag, New York, 1984.

[4] E. Hanamura, Solid State Science, vol. 124, Springer-Verlag, New York, 1996, Relaxation of Excited State and Photo-induced Phase Transitions, K. Nasu (ed.).

[5] W. Neumann and H. Sixl, Chem. Phys. 50 (1980), 273.

[6] W. Neumann and H. Sixl, Chem. Phys. 58 (1980), 273.

[7] C. Itoh, T. Kondoh, and K. Tanimura, Chem. Phys. Lett. 261 (1996), 191.

[8] H. Gross and H. Sixl, Chem. Phys. Lett. 91 (1982), 262.

[9] C. Itoh, T. Kondoh, and K. Tanimura, to be published. 
[10] K. S. Song and R. T. Williams (eds.), Solid State Science, vol. 105, Springer-Verlag, New York, 1992, Self-trapped excitons.
[11] C. Itoh, T. Kondoh, and K. Tanimura, J. Phys. Soc. Jpn (1999), in press. 


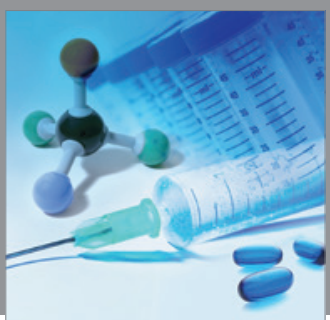

International Journal of

Medicinal Chemistry

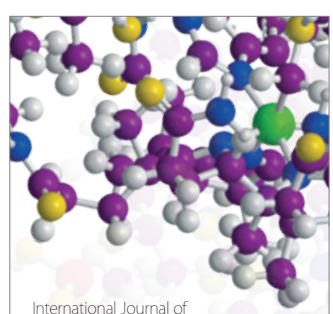

Carbohydrate Chemistry

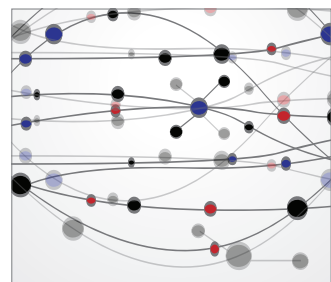

The Scientific World Journal
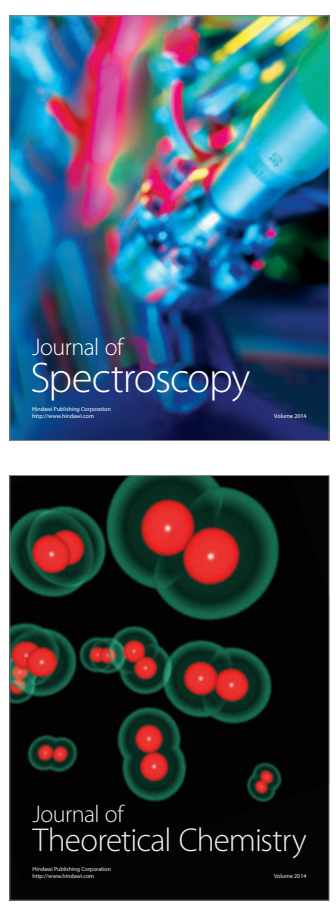
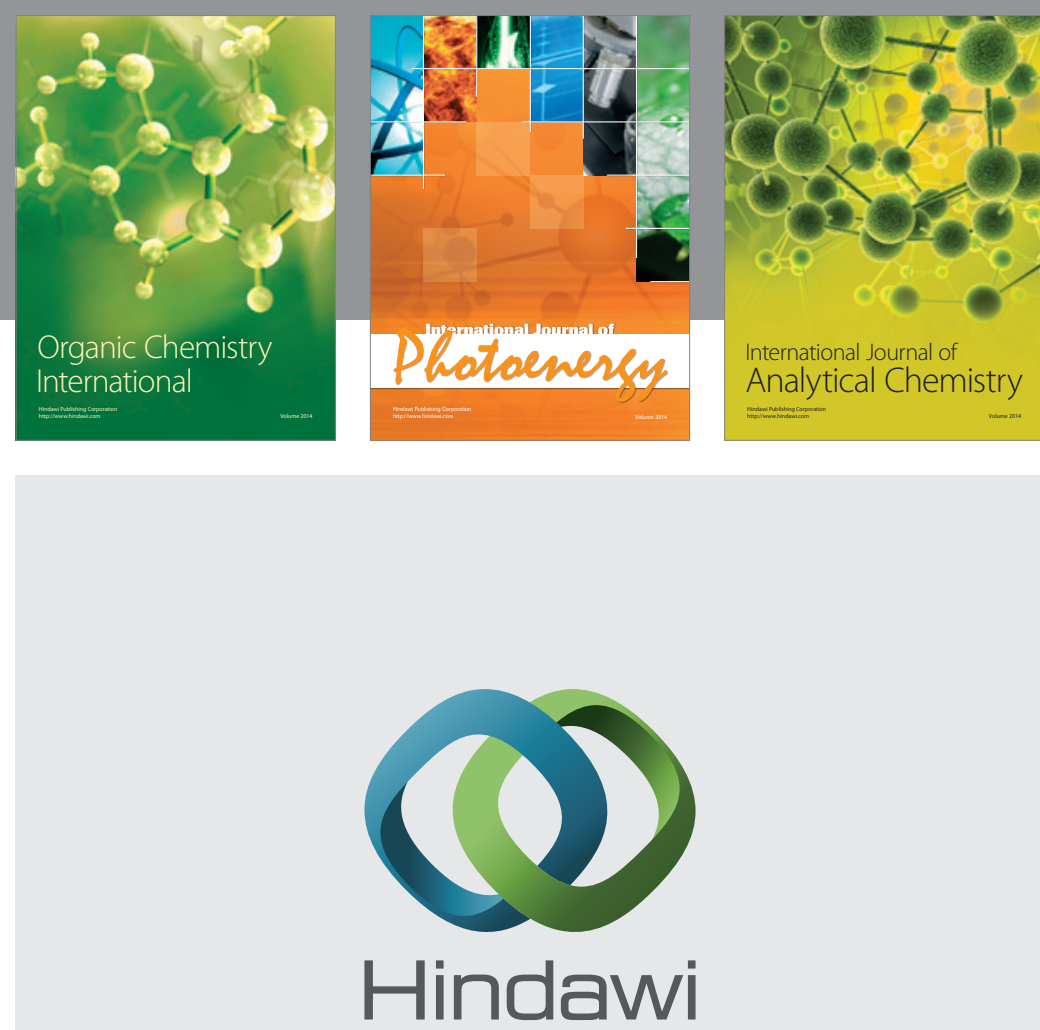

Submit your manuscripts at

http://www.hindawi.com
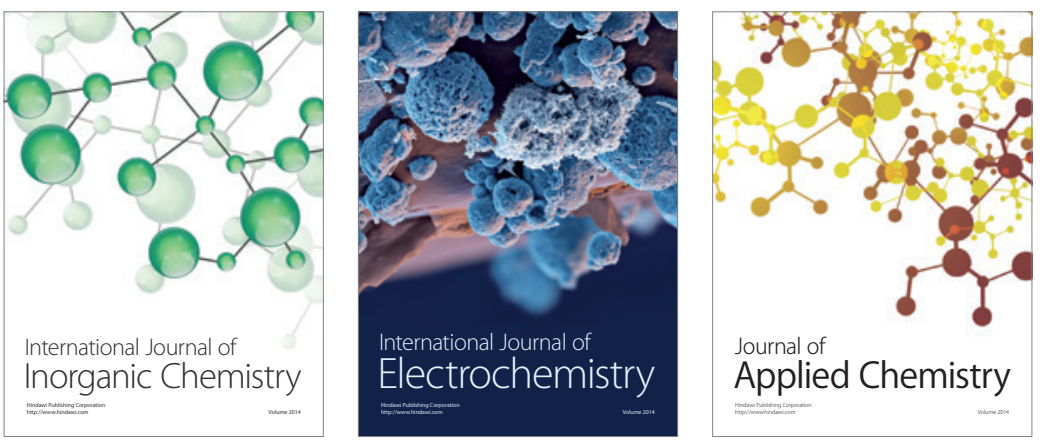

Journal of

Applied Chemistry
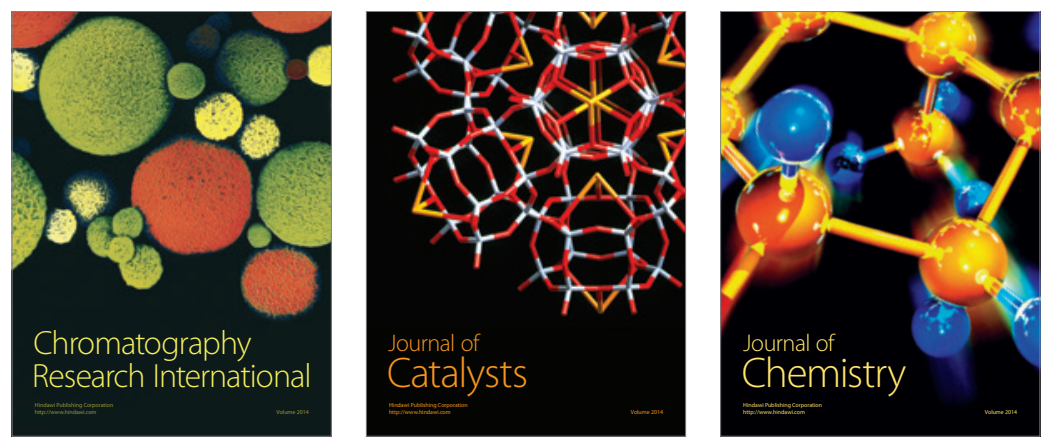
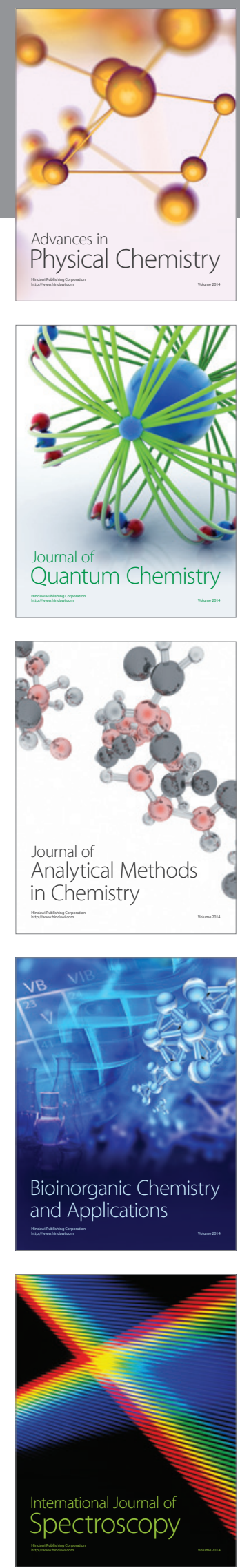